\title{
Multiple Courses of Rituximab Produce Sustained Clinical and Radiographic Efficacy and Safety in Patients with Rheumatoid Arthritis and an Inadequate Response to 1 or More Tumor Necrosis Factor Inhibitors: 5-Year Data from the REFLEX Study
}

\author{
EDWARD C. KEYSTONE, STANLEY B. COHEN, PAUL EMERY, JOEL M. KREMER, MAXIME DOUGADOS, \\ JAMES E. LOVELESS, CAROL CHUNG, PAMELA WONG, PATRICIA B. LEHANE, and HELEN TYRRELL
}

ABSTRACT. Objective. This 5-year observational posthoc analysis of the REFLEX study and its open-label extension assessed clinical efficacy, radiographic response, and safety of rituximab (RTX) in patients with rheumatoid arthritis (RA) who had an inadequate response to tumor necrosis factor (TNF) inhibitors.

Methods. Patients in REFLEX were originally randomized to placebo (PBO) + methotrexate (MTX; PBO-randomized) or RTX + MTX (RTX-randomized). PBO-randomized patients were rescued with RTX as appropriate. Patients responding to initial RTX treatment could receive further RTX courses. For clinical efficacy and safety analyses, PBO-randomized patients were re-baselined prior to first RTX treatment and the data were pooled with RTX-randomized patient data. Efficacy outcomes 24 weeks after each course were calculated relative to first RTX pretreatment baseline. Radiographic outcomes were assessed relative to randomization baseline for both PBO-randomized and RTX-randomized groups.

Results. A total of 480 patients received $\geq 1$ RTX course. At 24 weeks, American College of Rheumatology 20/50/70 responses were $62.0 \%, 30.8 \%$, and $13.0 \%$, respectively at course 1 ( $\mathrm{n}=$ $400)$ and $70.3 \%, 41.8 \%$, and $22.0 \%$ at course $5(\mathrm{n}=91)$. European League Against Rheumatism good/moderate responses were $77.2 \%$ and $84.4 \%$ at courses $1(n=390)$ and $5(n=90)$. Rates of adverse events (AE), serious AE, and infections generally remained stable. Rate of progressive joint damage (PJD; change in mean Total Sharp Score) decreased over time in both PBO-randomized $(\mathrm{n}=79)$ and RTX-randomized $(\mathrm{n}=105)$ groups. Mean change from baseline in PJD over 5 years was greater in PBO-randomized versus RTX-randomized patients (5.51 vs 3.21).

Conclusion. RTX re-treatment over 5 years is associated with maintained or improved efficacy, continued inhibition of PJD, and a safety profile consistent with that previously reported. A delay in initiating RTX treatment may result in increased PJD. (First Release Oct 1 2012; J Rheumatol 2012;39:2238-46; doi:10.3899/jrheum.120573)

Key Indexing Terms:

RHEUMATOID ARTHRITIS BIOLOGICAL THERAPY CD20 ANTIBODY RITUXIMAB

From Mount Sinai Hospital and the University of Toronto, Toronto, Ontario, Canada; Metroplex Clinical Research Center, Dallas, Texas, USA; the University of Leeds and Leeds Teaching Hospitals, Leeds, UK; Albany Medical College, Albany, New York, USA; René Descartes University, Paris, France; St. Luke's Rheumatology, Boise, Idaho, USA; Genentech Inc., South San Francisco, California, USA; and Roche Products Limited, Welwyn Garden City, UK.

Supported by F. Hoffmann-La Roche Ltd. Dr. Keystone has received grants/research support from and acted as a consultant for Abbott, AstraZeneca, BMS, Centocor, Roche, Merck, Pfizer and UCB, has received grants/research support from Amgen, Genzyme and Novartis, and has acted as a consultant for Biotest, Genentech and Nycomed. Dr. Cohen has received grants/research support from and acted as a consultant for Amgen, BMS, Roche, Merck and Pfizer, has received grants/research support from Abbott, AstraZeneca, Centocor, Genzyme, Novartis and $U C B$, and has acted as a consultant for Genentech. Dr. Emery has acted as a consultant for Pfizer, Merck, Abbott, BMS, Roche, Novartis, and $U C B$. Dr. Kremer has received grants/research support from Genentech

\author{
and Roche, and has acted as a consultant and on speakers' bureau for \\ Genentech. Dr. Dougados has received grants/research support from and \\ acted as a consultant for Roche, Abbott, Pfizer, BMS, UCB, Novartis, and \\ Merck. Dr. Loveless has acted as a consultant and on speakers' bureau \\ for Roche. \\ E.C. Keystone, MD, FRCPC, Director, The Rebecca MacDonald Centre \\ for Arthritis and Autoimmune Disease, Mount Sinai Hospital, and \\ Professor of Medicine, University of Toronto; S.B. Cohen, MD, Medical \\ Director, Metroplex Clinical Research Center; P. Emery, MA, MD, FRCP, \\ Clinical Director, University of Leeds and Leeds Teaching Hospitals; \\ J.M. Kremer, MD, Rheumatologist, Albany Medical College; \\ M. Dougados, MD, Professor of Rheumatology, René Descartes \\ University; J.E. Loveless, MD, Principal Investigator, St. Luke's \\ Rheumatology; C. Chung, PhD, Associate Director, Biostatistics; \\ P. Wong, MPh, Biostatistician, Genentech Inc.; P.B. Lehane, PhD, BSc \\ (Hons), Clinical Scientist; H. Tyrrell, BSc, Senior Clinical Scientist, \\ Roche Products Ltd.
}

Address correspondence to Dr. E.C. Keystone, Rebecca MacDonald Personal non-commercial use only. The Journal of Rheumatology Copyright @ C 2012. All rights reserved. 
Centre for Arthritis and Autoimmune Diseases, Mount Sinai Hospital, The Joseph and Wolf Lebovic Building, 2nd Floor, Room 2-006, Box 4, 60 Murray Street, Toronto, Ontario M5G 1X5, Canada.

E-mail:edkeystone@mtsinai.on.ca

Full Release Article. For details see Reprints/Permissions at jrheum.org Accepted for publication July 23, 2012.

Rituximab (RTX), a monoclonal antibody that selectively targets CD20-positive B cells, is indicated for the treatment of moderate to severely active rheumatoid arthritis (RA) in patients with an inadequate response or intolerance to $\geq 1$ tumor necrosis factor inhibitors (TNF-IR patients) ${ }^{1}$. In the phase III REFLEX study (Randomized Evaluation of Long-Term Efficacy of Rituximab in RA) conducted in TNF-IR patients with active RA, a single course of RTX in combination with methotrexate (MTX) was shown to significantly improve disease activity 24 weeks following treatment compared with placebo plus MTX $^{2}$. Further analyses of REFLEX demonstrated that RTX + MTX treatment significantly inhibited progression of joint damage versus placebo + MTX at 56 weeks $^{3}$ and at 2 years ${ }^{4}$. A pooled analysis of longterm clinical efficacy in TNF-IR patients from phase II and III studies within the RTX RA development program has demonstrated that repeated treatment with up to 5 courses of RTX led to sustained levels of efficacy, with no unexpected safety findings and consistent rates of infection and serious infection $^{5}$. In addition, a recent pooled, longterm safety analysis of all clinical trials within the RTX RA clinical program over 9.5 years (3194 patients with 11,962 patient-years of observation), including MTX-naive, disease-modifying antirheumatic drug inadequate responder (DMARD-IR) and TNF-IR patients, confirmed RTX in combination with MTX remains generally well tolerated over time and multiple courses, with a safety profile similar to that of placebo and consistent with published data on patients with RA ${ }^{5 a}$. Rates of adverse events (AE), serious adverse events (SAE), and serious infectious events (SIE) were comparable across patient populations, and these findings indicated that there was no evidence of an increased safety risk over time or increased reporting rates of any types of AE with prolonged exposure to RTX during 9.5 years of observation.

Current RA guidelines recommend early aggressive treatment with a goal of clinical remission or low disease activity (LDA), because this approach is associated with better longterm structural and functional outcomes for patients ${ }^{6,7}$. Given the association between structural damage and longterm loss of function ${ }^{8}$, maintaining inhibition of joint damage over time is critical. Data illustrating improvements in progression of joint damage have been presented regarding patients with early RA treated with nonbiologic DMARD combinations and in early RA and DMARD-IR patients with biologics $9,10,11$. However, no biologic RA therapy other than RTX has been shown to retard or inhibit joint damage in TNF-IR patients.

The purpose of our current analysis was to assess the clinical efficacy, radiographic response, and safety of RTX in the TNF-IR patient population from REFLEX and its longterm extension when administered on an "as-needed" basis over 5 years, and to explore the effect of a delay in the initiation of RTX treatment.

\section{MATERIALS AND METHODS}

Patients, study design, and treatment. REFLEX was a multicenter, randomized, double-blind, placebo-controlled clinical trial conducted in patients with an inadequate response to 1 or more TNF inhibitors ${ }^{2}$. The inclusion criteria have been described in detail ${ }^{2}$. In brief, patients were required to have (1) an RA diagnosis [according to revised 1987 American College of Rheumatology (ACR) criteria]; (2) received MTX for $\geq 12$ weeks at 10-25 mg/week and at a stable dose for $\geq 4$ weeks prior to screening; (3) both swollen joint count (SJC; 66 joints) and tender joint count (TJC; 68 joints) $\geq 8$ at screening and randomization; and (4) C-reactive protein $\geq 1.5 \mathrm{mg} / \mathrm{dl}$ or erythrocyte sedimentation rate $(\mathrm{ESR}) \geq 28 \mathrm{~mm} / \mathrm{h}$.

Patients were randomly allocated (3:2) to treatment with RTX $2 \times 1000$ mg or placebo given as intravenous (IV) infusions 2 weeks apart, with all infusions premedicated with IV methylprednisolone $100 \mathrm{mg}$. Oral prednisone was administered at $60 \mathrm{mg} /$ day on Days 2-7 and at $30 \mathrm{mg} /$ day on Days 8-14. Following analysis of concomitant use of corticosteroids in the DANCER study ${ }^{12}$, the corticosteroid regimen for the REFLEX extension protocol was amended on July 27, 2006, to no longer include the oral prednisone regimen for subsequent treatment courses. Patients continued to receive concomitant MTX 10-25 $\mathrm{mg} /$ week at a stable dose and were permitted to receive stable background doses of oral corticosteroids $(\leq 10$ mg/day prednisolone or equivalent) and nonsteroidal antiinflammatory drugs throughout. Patients originally randomized to placebo who did not respond to the initial treatment course (i.e., $<20 \%$ improvement in SJC and TJC) could receive rescue therapy with RTX from Week 16.

Patients were required to demonstrate response to initial RTX treatment to be eligible for further treatment courses in the open-label extension (OLE), including patients originally randomized to placebo and rescued with RTX. Repeat treatment was administered on an as-needed basis if both SJC and TJC were $\geq 8$ and at the discretion of the treating physician in the OLE study. The minimum re-treatment interval was 24 weeks following course 1 and 16 weeks for course 2 and all subsequent courses.

REFLEX and its OLE were conducted in accordance with the ethical principles of the Declaration of Helsinki and adhered to the principles outlined in the Good Clinical Practice ICH Tripartite Guideline (January 1997).

Assessments. This was an observational, posthoc analysis of the REFLEX study and its OLE from baseline to 5 years and was open-label from the point of administration of the second course of treatment.

Clinical efficacy. Clinical efficacy was analyzed in all patients who received at least 1 course of RTX (including those originally randomized to placebo who received RTX as rescue therapy). Patients originally randomized to placebo were re-baselined prior to their first RTX treatment; for this analysis, placebo data were pooled with RTX patient data from time of first RTX treatment. These patients are referred to here as the RTX-treated population.

Assessments of clinical outcomes included ACR responses, European League Against Rheumatism (EULAR) responses, and Disease Activity Score in 28 joints using ESR (DAS28-ESR), LDA, and remission (defined as DAS28-ESR $\leq 3.2$ and $<2.6$, respectively). Physical function was assessed using the Health Assessment Questionnaire-Disability Index (HAQ-DI).

Outcomes are presented as observed data either 24 weeks following each 
course or continuously over time irrespective of number of courses received during the 5-year (240-week) observation period. A sensitivity analysis [completers analysis: within-patient within-visit (WW)] was conducted for DAS28-ESR LDA and remission, which included only those patients with data available 24 weeks after each treatment course for 5 courses. The observed value closest to the timepoint of interest $( \pm 4$ weeks) for each component (e.g., TJC) was used to calculate the composite scores (e.g., DAS). No further imputations were made for missing composite scores.

Radiographic efficacy. Radiographic data are presented for a subgroup of patients who had radiographs available at baseline and Year 5. The data were assessed relative to the initial randomization baseline for both groups (patients originally randomized to placebo and patients originally randomized to RTX). Assessments included changes in Genant-modified Total Sharp Score ${ }^{13}$ (mTSS), erosion score, and joint space narrowing over time, annualized rates of progression, and the proportion of patients with no radiographic progression (defined as change in $\mathrm{mTSS} \leq 0$ ). Missing radiographic data were imputed for Year 4 only using linear extrapolation of the progression observed from baseline to the last value prior to the missing value. Analyses of annualized progression rates were based on available cases without imputation for missing data.

Safety. Safety was assessed in the RTX-treated population including patients originally randomized to placebo and subsequently rescued with RTX. All AE after the first RTX treatment and up to 5 years (240 weeks) were graded and recorded using the Medical Dictionary for Regulatory Activities (MedDRA, version 13.1). SAE were defined as events that were fatal, immediately life-threatening, required inpatient hospitalization or prolongation of an existing hospitalization, were medically significant, or required intervention to prevent one of the above outcomes. The rate of infections and SIE were also recorded, with the latter defined as infections that met the definition of an SAE and/or required treatment with IV antiinfectives.

\section{RESULTS}

Patient demographics and disease characteristics, disposition, and re-treatment interval. A total of 517 patients were randomized and received study medication in REFLEX (308 patients to RTX + MTX, and 209 to placebo + MTX).
Subsequently, 480 patients (308 originally randomized to RTX and 172 originally randomized to placebo) transferred to the OLE study, where they may have received further treatments with RTX. The RTX-treated population for this analysis comprised 480 patients who received at least 1 course of RTX. Patient demographics and baseline disease characteristics in the RTX-treated population are shown in Table 1 . The majority of patients were female $(81 \%)$. The mean age was 52.5 years. At baseline, patients had longstanding RA (mean $12 \mathrm{yrs}$ ) with evidence of active disease (mean DAS28-ESR 6.9, mean SJC 24, and mean TJC 35).

The numbers of patients receiving subsequent RTX courses were 317 ( $\geq 2$ courses), 259 ( $\geq 3$ courses), 195 ( $\geq 4$ courses), and 122 ( $\geq 5$ courses; Figure 1). The greatest number of withdrawals occurred following the first course of treatment [133 patients $(27.7 \%)$, principally for non-safety reasons. This may reflect the requirement for response to the first course to be eligible for re-treatment. The withdrawal rate was about $10 \%$ for subsequent courses. Withdrawals due to AE were low [29 patients (6\%) withdrew because of AE over 5 RTX courses], with most occurring during the first course (Figure 1). Over the 5 years, the most common AE leading to withdrawal were related to the following MedDRA system organ classes: general disorders and administration-site conditions (7 events, all infusion-related reactions), neoplasms and malignancies ( 7 events), infections and infestations ( 6 events), and musculoskeletal and connective tissue disorders (4 events, all RA exacerbation).

Patients received up to 12 courses of RTX over 5 years. Median treatment intervals among patients with repeat courses were 0.98 years (courses $1-2 ; \mathrm{n}=317$ ), 1.06 years

Table 1. Patient demographics and baseline characteristics. Baseline is compared to first rituximab (RTX) treatment baseline (RTX-treated population) or initial randomization baseline (placebo-randomized and RTX-randomized populations).

\begin{tabular}{lccc}
\hline & & \multicolumn{2}{c}{ Radiographic Population } \\
Characteristic & RTX-treated, & Placebo-randomized, & RTX-randomized, \\
& $\mathrm{n}=480$ & $\mathrm{n}=79$ & $\mathrm{n}=105$ \\
\hline Female, $\mathrm{n}(\%)$ & $387(80.6)$ & $62(78.5)$ & $89(84.8)$ \\
Race, white, $\mathrm{n}(\%)$ & $422(87.9)$ & $69(87.3)$ & $89(84.8)$ \\
Age, yrs, mean (SD) & $52.5(12.4)$ & $52.9(10.7)$ & $53.5(10.7)$ \\
RA disease duration, yrs, mean (SD) & $12.0(8.1)$ & $10.5(7.4)$ & $13.5(9.4)$ \\
RF+ and/or ACPA+, n/N (\%) & $393 / 437(89.9)$ & $72 / 74(97.3)$ & $91 / 96(94.8)$ \\
TJC, 68 joints, mean (SD) & $34.7(15.6)$ & $34.2(16.3)$ & $32.6(13.7)$ \\
SJC, 66 joints, mean (SD) & $23.6(12.3)$ & $24.5(13.3)$ & $23.9(11.4)$ \\
CRP, mg/dl, mean (SD) & $3.8(4.0)$ & $3.8(3.3)$ & $3.4(3.5)$ \\
ESR, mm/h, mean (SD) & $47.3(26.1)$ & $49.6(25.3)$ & $46.5(23.9)$ \\
DAS28-ESR, mean (SD) & $6.9(1.0)$ & $6.9(1.0)$ & $6.8(1.0)$ \\
HAQ-DI, mean (SD) & $1.9(0.6)$ & $1.9(0.5) *$ & $1.8(0.6)$ \\
Baseline mTSS, mean (SD) & - & $30.9(33.8)$ & $28.4(23.6)$ \\
\hline
\end{tabular}

$* \mathrm{n}=78$. RA: rheumatoid arthritis; ACPA: anticitrullinated protein antibodies; CRP: C-reactive protein; DAS28: Disease Activity Score in 28 joints; ESR: erythrocyte sedimentation rate; HAQ-DI: Health Assessment Questionnaire Disability Index; mTSS: modified Total Sharp Score; RF: rheumatoid factor; SJC: swollen joint count; TJC: tender joint count. 


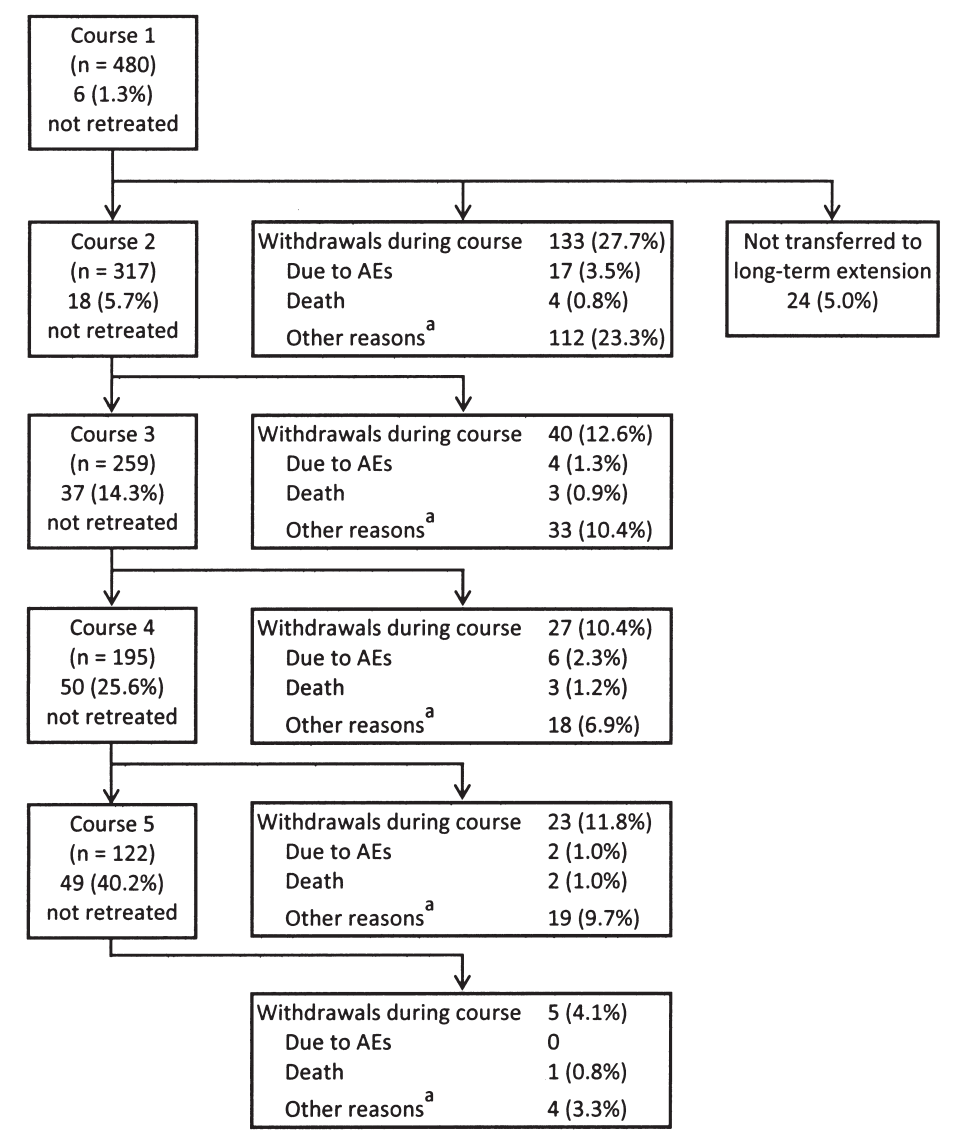

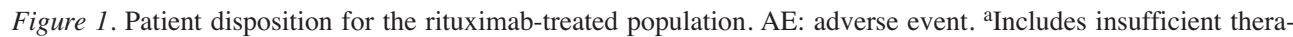
peutic response, failure to return, protocol violation, refused treatment, physician's decision, administration/other.

(courses $2-3 ; \mathrm{n}=259), 1.02$ years (courses $3-4 ; \mathrm{n}=195)$, and 0.91 years (courses $4-5 ; n=122$ ).

Clinical efficacy. Clinical efficacy outcomes at 24 weeks following each RTX course compared with first RTX baseline are summarized in Table 2. Improvements in signs and symptoms of RA were sustained or improved over 5 courses of RTX treatment, as measured by ACR response rates and EULAR response rates. Efficacy outcomes for subsequent treatments beyond course 5 were maintained (data not shown); decreasing patient numbers limit the conclusions that can be drawn regarding these data.

The proportion of patients achieving DAS28-LDA increased consistently with each course to course 3 and was then maintained, and the proportion achieving DAS28 remission increased to course 4 and was then maintained. A sensitivity analysis of patients with data available at 24 weeks after each of 5 courses (WW analysis; $n=72$ ) showed a consistent increase in DAS28-LDA course on course (Table 2), while DAS28 remission rates increased to course 4. When considered as a continuous change over time from first RTX treatment (regardless of number of courses received), the mean DAS scores decreased rapidly between baseline and Week 16 and continued to decrease minimally over time (Figure 2).

RTX treatment also resulted in an initial improvement in physical function followed by maintenance course on course (Table 2). In addition, the proportion of patients achieving a minimal clinically important difference (MCID) in HAQ-DI (decrease of $\geq 0.22$ ) was maintained over multiple courses $-66.0 \%$ after course 1 and $71.1 \%$ after course 5 (Table 2).

Radiographic analyses. Radiographic analyses were conducted on a subgroup of patients who had radiographs available at baseline and Year 5. This population comprised 184 patients, of whom 105 and 79 were originally randomized to RTX and placebo, respectively. Among the 79 placebo-randomized patients, 71 were rescued with RTX before Year 1, 5 between years 1 and 2, and 1 between years 2 and 3;2 patients did not receive rescue therapy. Baseline demographics and disease characteristics were balanced between the 2 groups (Table 1), with the exception of RA disease duration, which was slightly longer in the RTX-randomized group (13.5 years) than in the placebo-randomized group (10.5 years).

Progressive joint damage (PJD) increased in both groups 
Table 2. Efficacy outcomes at 24 weeks by course. All change assessments are relative to baseline of first rituximab (RTX) treatment.

\begin{tabular}{lccccc}
\hline & \multicolumn{4}{c}{ RTX-treated Population, $\mathrm{n}=480$} \\
& 1st Course & 2nd Course & 3rd Course & 4th Course & 5th Course \\
\hline ACR responses, $\mathrm{n}$ & 400 & 279 & 225 & 161 & 91 \\
ACR20, $\%$ & 62.0 & 72.8 & 72.4 & 65.8 & 70.3 \\
ACR50, $\%$ & 30.8 & 41.2 & 47.6 & 44.7 & 41.8 \\
ACR70, $\%$ & 13.0 & 19.4 & 26.2 & 24.2 & 22.0 \\
EULAR responses, $\mathrm{n}$ & 390 & 275 & 224 & 157 & 90 \\
Moderate/good response, \% & 77.2 & 89.5 & 88.8 & 91.7 & 84.4 \\
DAS28 LDA, \% & 16.9 & 26.5 & 35.3 & 31.2 & 28.9 \\
DAS28 remission, $\%$ & 8.7 & 14.2 & 18.3 & 19.1 & 13.3 \\
Within-patient within-visit sensitivity analysis & & & & \\
No. patients & 72 & 72 & 72 & 72 & 72 \\
DAS28 LDA, $\%$ & 12.5 & 15.3 & 22.2 & 23.6 & 30.6 \\
DAS28 remission, \% & 5.6 & 8.3 & 11.1 & 15.3 & 12.5 \\
DAS28-ESR, n & 390 & 275 & 224 & 157 & 90 \\
Mean (SD) change & $-2.19(1.42)$ & $-2.71(1.35)$ & $-2.99(1.44)$ & $-2.99(1.55)$ & $-3.04(1.70)$ \\
HAQ-DI, $n$ & 400 & 283 & 224 & 161 & 90 \\
Mean (SD) change & $-0.48(0.57)$ & $-0.52(0.57)$ & $-0.55(0.60)$ & $-0.55(0.61)$ & $-0.61(0.64)$ \\
Patients (\%) with an MCID HAQ-DI decrease of $\geq 0.22$ & & & \\
& 66.0 & 68.6 & 70.1 & 69.6 & 71.1 \\
\hline
\end{tabular}

LDA (low disease activity) defined as DAS28-ESR (Disease Activity Score in 28 joints using erythrocyte sedimentation rate) $\leq 3.2$ and remission defined as DAS28-ESR < 2.6. ACR: American College of Rheumatology; EULAR: European League Against Rheumatism; HAQ-DI: Health Assessment Questionnaire-Disability Index; MCID: minimal clinically important difference.

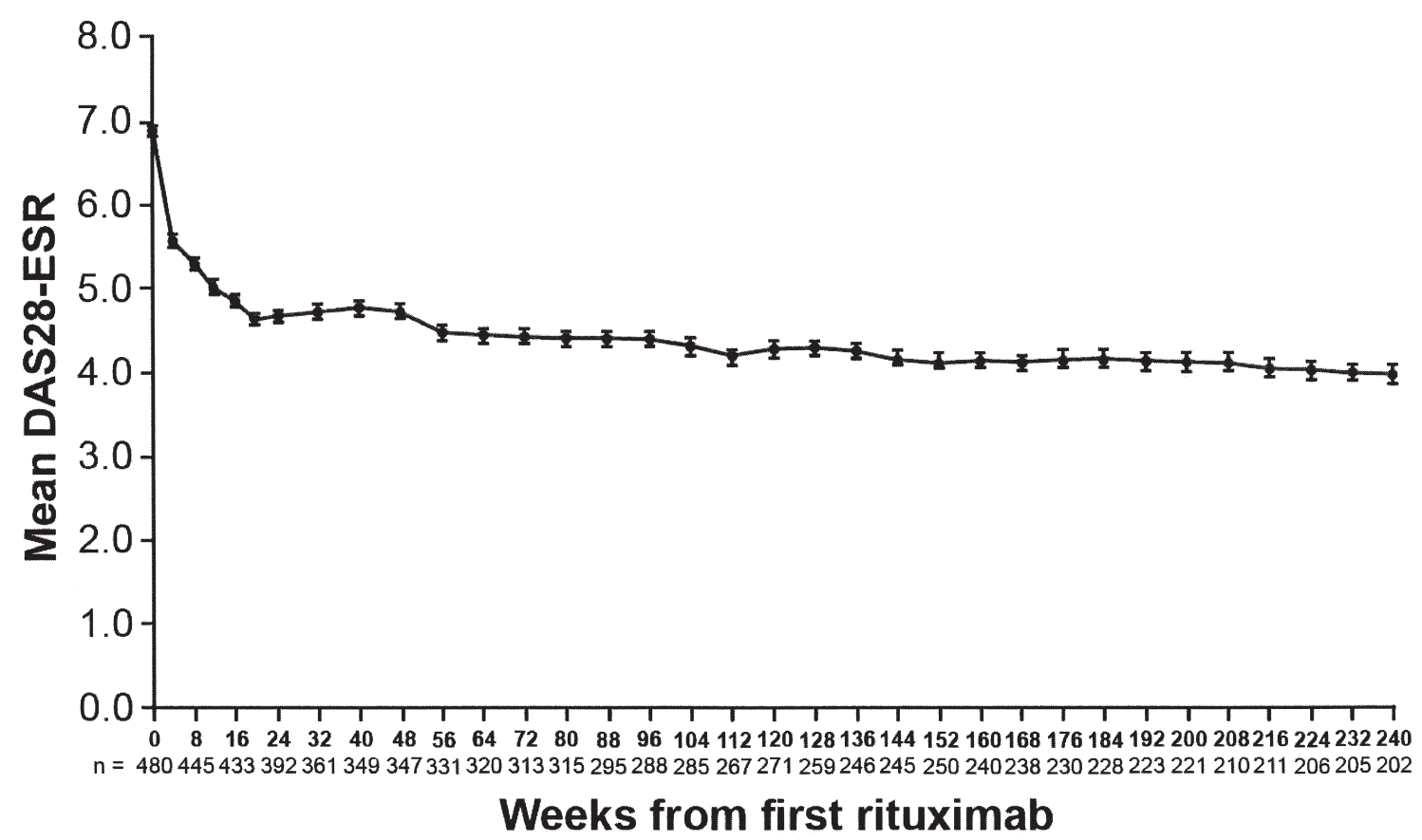

Figure 2. Continuous mean 28-joint Disease Activity Score-erythrocyte sedimentation rate (DAS28-ESR) by study visit in the rituximab-treated population. Vertical bars are \pm 1 standard error. At Week 4, n =362; Week 12, n= 439; Week 20, $\mathrm{n}=395$.

over time to 5 years (Table 3). However, overall radiographic damage remained numerically higher in patients originally randomized to placebo, the majority of whom
(90\%) subsequently switched to RTX within the first year, compared with those originally randomized to RTX. The mean (SE) change in mTSS from baseline at Year 5 was 3.21 
Table 3. Change from baseline in modified Total Sharp Score (mTSS) over time.

\begin{tabular}{lccc}
\hline$\Delta$ mTSS, mean (SEM) & Year 1 & Year 4 & Year 5 \\
\hline PBO-randomized, $\mathrm{n}=79 *$ & $2.39(0.45)$ & $5.26(0.91)$ & $5.51(0.95)$ \\
RTX-randomized, $\mathrm{n}=105^{*}$ & $1.08(0.24)$ & $2.86(0.60)$ & $3.21(0.64)$ \\
\hline
\end{tabular}

* At Year 1, $\mathrm{n}=75$ (PBO-randomized); $\mathrm{n}=102$ (RTX-randomized). PBO: placebo; RTX: rituximab.

(0.64) for patients originally randomized to RTX and 5.51 (0.95) for those originally randomized to placebo (Table 3 ). Changes in erosion scores and joint space narrowing over time showed a pattern similar to that seen with mTSS (data not shown).

A numerically greater proportion of patients showed no radiographic progression (change in mTSS $\leq 0$ ) from baseline to Year 5 in the RTX-randomized group compared with the placebo-randomized group (32.4\% vs $21.5 \%)$. This difference was also evident when measured at Year $1(48.0 \%$ vs $32.0 \%)$ and at Year 4 (32.4\% vs $24.1 \%)$. In both groups, the proportion of patients with no progression was maintained between years 4 and 5 .

The annualized rate of progression of mTSS decreased in both groups over time. Rates were numerically lower in patients originally randomized to RTX than in patients originally randomized to placebo between baseline and Year 1 (0.91 vs 2.08 TSS units) and as estimated between years 1 and 4 (0.56 vs 0.89 TSS units). Between years 4 and 5, RTX-randomized patients showed rates of progression similar to those originally randomized to placebo ( $0.33 \mathrm{vs}$ 0.25 TSS units, respectively).

Safety. Over the 5 years, rates of AE, SAE, and infections did not increase and generally remained stable (Figure 3 ) in the RTX-treated population (1768 patient-years). The overall rates per 100 patient-years were 344.87 (95\% CI 336.32-353.64) for AE and 22.34 (95\% CI 20.24-24.65) for SAE. AE rates were highest during the year after first RTX exposure (owing in part to infusion-related reactions during first infusion of the first course), declined in the second year, and generally remained stable thereafter, irrespective of number of courses received. The overall rate of all infections was 97.50 per 100 patient-years $(95 \%$ CI 93.01-102.21) with an overall SIE rate of 5.60 per 100 patient-years (95\% CI 4.60-6.82). The SIE rate was highest in the 2 years following first RTX exposure and decreased in subsequent years. The most common SIE was pneumonia, which was reported in 5 patients $(1 \%)$ during course 1 , and in $1(<1 \%), 4(2 \%), 0$, and $1(<1 \%)$ patients during courses 2, 3, 4, and 5, respectively. Serious opportunistic infections were rare, with 1 event (Scedosporium lung infection) reported in an RTX-treated patient during the 5 -year observation period. Two further serious opportunistic infections were reported after the 5-year cutoff: 1 case of pharyngeal abscess (organism unspecified), and a previously reported case of progressive multifocal leukoencephalopathy with fatal outcome ${ }^{14}$. No cases of pulmonary tuberculosis (TB), extrapulmonary TB, atypical mycobacterial infection, or multidrug-resistant TB were observed in this cohort of patients. No cases of hepatitis B reactivation were reported. A single case of de novo hepatitis B infection occurred in a 59-year-old female patient following a dental procedure, as reported ${ }^{2}$.

Infusion-related reactions (IRR) were reported in 189 RTX-treated patients (39\%). The frequency, type, and intensity of IRR and other AE were consistent with that reported $^{5 a}$. Serious IRR were rare and included anaphylactic or anaphylactoid reactions, chest pain, headache, and tachycardia. Seven events were reported in 6 patients $(1 \%)$, of which 5 occurred during the first infusion of a course.

There were 19 fatal AE in RTX-treated patients. Causes of death varied and were typical for a patient population with refractory active RA. Most common primary causes of death included neoplasms (benign, malignant, and unspecified; $n=6)$ and cardiac disorders $(n=5)$. Incidence of death did not appear to increase with cumulative exposure to RTX and there were no infusion-related deaths.

\section{DISCUSSION}

Our analysis of TNF-IR patients in the REFLEX study demonstrates that RTX repeat treatment leads to clinical efficacy responses that are either maintained or continue to improve over the 5-year period. RTX repeat treatment was also associated with sustained radiographic outcomes, as shown by the continued inhibition of PJD over 5 years. The safety profile was consistent with previous analyses of the longterm safety of RTX across multiple RA populations. To our knowledge this is the first comprehensive, longterm analysis of the efficacy and safety of a biologic therapy in a TNF-IR, refractory RA population.

Maintained or improved efficacy responses with RTX treatment over 5 years were seen using both ACR and EULAR response measures. In addition, there was evidence of an increase in the proportion of patients achieving clinically important levels of disease activity (LDA and remission, as measured by DAS28-ESR) with RTX repeat treatment over time, a finding that was supported by a sensitivity analysis (WW or "completers" analysis). Although this dataset included patients who received $>5$ courses of RTX over the 5 years, the associated patient numbers were low and it was therefore difficult to draw firm conclusions regarding the efficacy of RTX beyond 5 courses.

Studies have demonstrated that continual treatment with biologic agents is associated with sustained inhibition of radiographic progression, albeit in a less refractory patient population ${ }^{10,11}$. An earlier analysis of the REFLEX study also showed that RTX treatment led to sustained inhibition of PJD for up to 2 years $^{3,4}$. Our current analysis extends 
A
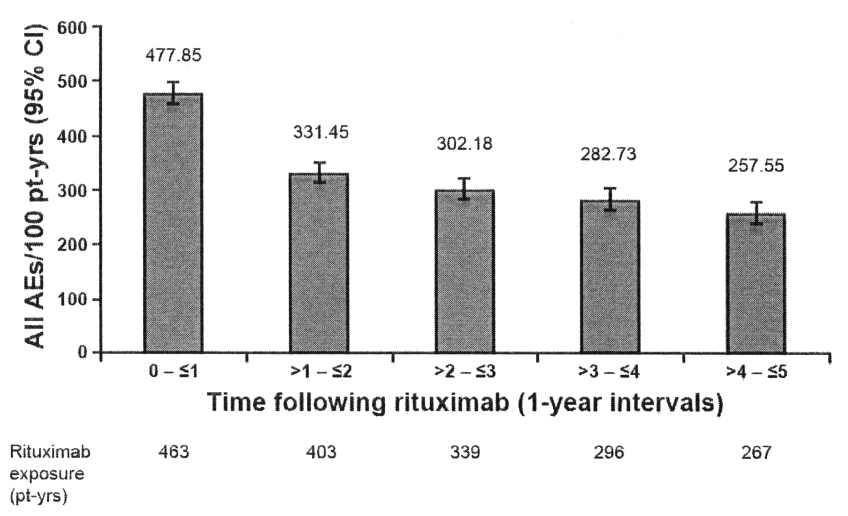

C

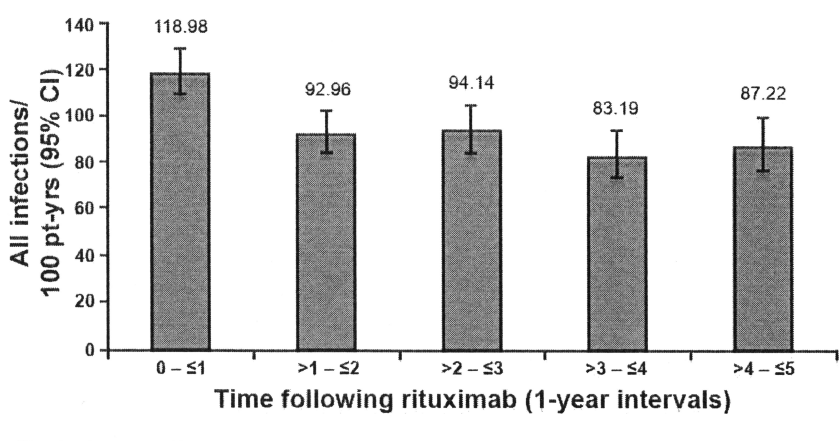

Rituximab

exposure
(pt-yrs)
B

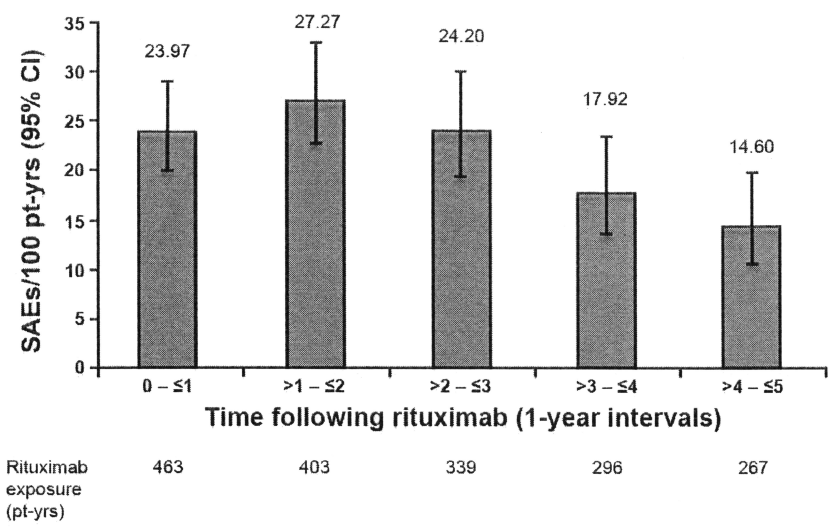

D

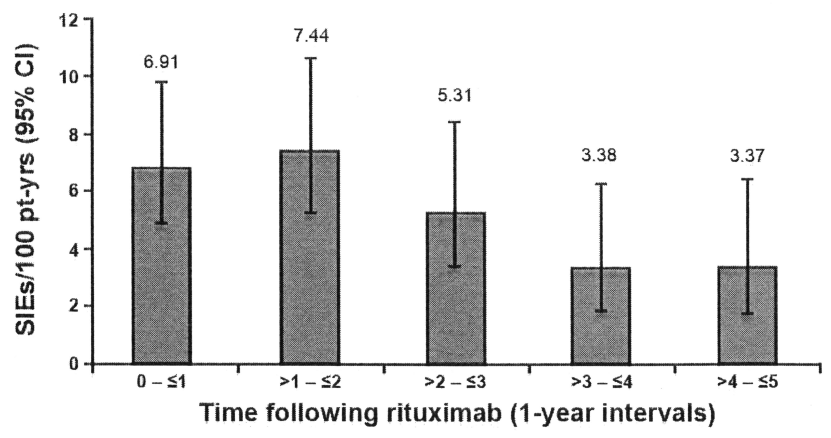

Rituximab 463 exposur

463

403

339

296

267

Figure 3. Rates of adverse events (AE), serious adverse events (SAE), and infections over time to 5 years (rituximab-treated population). Panel A, all adverse events; Panel B, serious adverse events; Panel C, all infections; and Panel D, serious infections (SIE). 1 year = 48 weeks. Pt-yrs: patient-years.

these observations, in this refractory patient population, with RTX repeat treatment now shown to continue to inhibit PJD for 5 years in patients continuing RTX. In addition, patients originally randomized to RTX had enhanced inhibition of PJD at 5 years compared with patients originally randomized to placebo and later rescued with RTX (and consequently experiencing a delay to onset of treatment of about 1 year). The proportion of patients with no radiographic progression was also greater in the group randomized to RTX at baseline. RTX patients were more likely to have no radiographic progression at Year 1, a difference that remained through years 4 and 5. Overall, these results suggest that even a relatively short delay in initiating RTX treatment, particularly in patients with disease refractory to conventional DMARD, may result in overall increased progression of joint damage.

The improvements over time in radiographic outcomes and RA signs and symptoms were observed alongside improvements in physical function, with mean HAQ-DI scores showing an initial improvement at course 1 , which was then maintained over 5 courses. Further, about $70 \%$ of patients at each course achieved an improvement in
HAQ-DI of at least the MCID of 0.22 points or greater at 24 weeks after each course compared with the pre-first RTX baseline.

The safety profile of RTX observed in the current analysis, following repeat treatment over 5 years, was consistent with that reported in the REFLEX TNF-IR RA population $^{2}$ and with a mixed RA population including MTX-naive, DMARD-IR, and TNF-IR patients ${ }^{5 \mathrm{a}}$. The rates of $\mathrm{AE}, \mathrm{SAE}$, and all infections remained generally stable over time out to 5 years. The rate of SIE decreased from the second year following first RTX exposure. A decrease in SIE rate over time is consistent with that reported for TNF inhibitors in patients enrolled in various national registries $^{15,16,17}$. The overall rate of withdrawals from RTX treatment was low throughout the 5-year observation period. Withdrawals due to AE were infrequent. Most withdrawals occurred during the first course and were for non-safety reasons, largely a result of the study design, which required response to the initial treatment. Thereafter the withdrawal rate was similar to that reported for other biologics $18,19,20$.

The mean interval between RTX courses in our analysis was longer than that included in current guidelines, which 
recommend considering repeat treatment after an interval of about 24 weeks using the treat-to-target approach in patients not achieving clinical remission or $\mathrm{LDA}^{21}$. Patients in REFLEX and its OLE were re-treated on an as-needed basis as judged by the physician, and this may have led to longer treatment intervals, with patients allowed to flare before treatment was repeated. More recent data show that a re-treatment frequency of about 6 months leads to an increase in efficacy ${ }^{22}$; treatment in accordance with this strategy has the potential to further improve longterm efficacy responses.

An important limitation of the findings is that this was a responder analysis (as observed data) and consequently, the outcomes reported are for those patients with an initial response to RTX who continued on RTX (or who started on placebo treatment and were later rescued with RTX). Data from course 2 onward are biased because only patients with a demonstrated response at course 1 were eligible for repeat treatment, and consequently comparisons of changes in efficacy from course 1 to subsequent timepoints should be treated with caution. In addition, the open-label design of the RTX repeat treatment study gives rise to the potential for physician and/or patient reporting bias. A further limitation is the low patient number available for the radiographic subgroup analysis. Data for this subgroup were available at years 1 and 4 but not for the intervening period; consequently, results represent mean changes in progression of joint damage for this 3-year period.

Our posthoc analysis of the REFLEX study confirms RTX as an effective longterm treatment option in this refractory RA population. RTX repeat treatment was associated with clinical efficacy and radiographic benefit out to 5 years, and a safety profile comparable with published longterm safety data on RTX and with safety data from other biologic agents in RA populations. A delay in the onset of RTX therapy may result in adverse radiographic outcomes.

\section{REFERENCES}

1. Dass S, Vital EM, Emery P. Rituximab: Novel B-cell depletion therapy for the treatment of rheumatoid arthritis. Expert Opin Pharmacother 2006;7:2559-70.

2. Cohen SB, Emery P, Greenwald MW, Dougados M, Furie RA, Genovese MC, et al. Rituximab for rheumatoid arthritis refractory to anti-tumor necrosis factor therapy: Results of a multicenter, randomized, double-blind, placebo-controlled, phase III trial evaluating primary efficacy and safety at twenty-four weeks. Arthritis Rheum 2006;54:2793-806.

3. Keystone E, Emery P, Peterfy CG, Tak PP, Cohen S, Genovese MC, et al. Rituximab inhibits structural joint damage in patients with rheumatoid arthritis with an inadequate response to tumour necrosis factor inhibitor therapies. Ann Rheum Dis 2009;68:216-21.

4. Cohen SB, Keystone E, Genovese MC, Emery P, Peterfy C, Tak PP, et al. Continued inhibition of structural damage over 2 years in patients with rheumatoid arthritis treated with rituximab in combination with methotrexate. Ann Rheum Dis 2010;69:1158-61.

5. Keystone EC, Fleischmann RM, Emery P, Dougados M, Williams $\mathrm{S}$, Linnik MD, et al. Multiple courses of rituximab produce sustained efficacy in patients with rheumatoid arthritis with an inadequate response to one or more TNF inhibitors [abstract]. Arthritis Rheum 2010;62 Suppl:S133.

5a. van Vollenhoven RF, Emery P, Bingham CO III, Keystone EC, Fleischmann R, Furst DE, et al. Long-term safety of rituximab in rheumatoid arthritis: 9.5-year follow-up of the global clinical trial programme with focus on adverse events of interest in RA patients. Ann Rheum Dis [in press].

6. Smolen JS, Aletaha D, Bijlsma JW, Breedveld FC, Boumpas D, Burmester G, et al. Treating rheumatoid arthritis to target: Recommendations of an international task force. Ann Rheum Dis 2010;69:631-7.

7. Smolen JS, Landewé R, Breedveld FC, Dougados M, Emery P, Gaujoux-Viala C, et al. EULAR recommendations for the management of rheumatoid arthritis with synthetic and biological disease-modifying antirheumatic drugs. Ann Rheum Dis 2010;69:964-75.

8. van der Heijde D, Landewe R, van Vollenhoven R, Fatenejad S, Klareskog L. Level of radiographic damage and radiographic progression are determinants of physical function: A longitudinal analysis of the TEMPO trial. Ann Rheum Dis 2008;67:1267-70.

9. Landewe RB, Boers M, Verhoeven AC, Westhovens R, van de Laar MA, Markusse HM, et al. COBRA combination therapy in patients with early rheumatoid arthritis: Long-term structural benefits of a brief intervention. Arthritis Rheum 2002;46:347-56.

10. van der Heijde D, Klareskog L, Landewe R, Bruyn GA, Cantagrel A, Durez P, et al. Disease remission and sustained halting of radiographic progression with combination etanercept and methotrexate in patients with rheumatoid arthritis. Arthritis Rheum 2007;56:3928-39.

11. van der Heijde D, Breedveld FC, Kavanaugh A, Keystone EC, Landewe R, Patra K, et al. Disease activity, physical function, and radiographic progression after longterm therapy with adalimumab plus methotrexate: 5-year results of PREMIER. J Rheumatol 2010;37:2237-46

12. Emery P, Fleischmann R, Filipowicz-Sosnowska A, Schechtman J, Szczepanski L, Kavanaugh A, et al. The efficacy and safety of RTX in patients with active rheumatoid arthritis despite methotrexate treatment: Results of a phase IIB randomized, double-blind, placebo-controlled, dose-ranging trial. Arthritis Rheum 2006;54:1390-400.

13. Genant HK, Jiang Y, Peterfy C, Lu Y, Redei J, Countryman PJ Assessment of rheumatoid arthritis using a modified scoring method on digitized and original radiographs. Arthritis Rheum 1998;41:1583-90.

14. Fleischmann RM. Progressive multifocal leukoencephalopathy following RTX treatment in a patient with rheumatoid arthritis Arthritis Rheum 2009;60:3225-8.

15. Askling J, Fored CM, Brandt L, Baecklund E, Bertilsson L, Feltelius N, et al. Time-dependent increase in risk of hospitalisation with infection among Swedish RA patients treated with TNF antagonists. Ann Rheum Dis 2007;66:1339-44.

16. Galloway JB, Hyrich KL, Mercer LK, Dixon WG, Fu B, Ustianowski AP, et al. Anti-TNF therapy is associated with an increased risk of serious infections in patients with rheumatoid arthritis especially in the first 6 months of treatment: Updated results from the British Society for Rheumatology Biologics Register with special emphasis on risks in the elderly. Rheumatology 2011;50:124-31.

17. Strangfeld A, Eveslage M, Schneider M, Bergerhausen HJ, Klopsch T, Zink A, et al. Treatment benefit or survival of the fittest: What drives the time-dependent decrease in serious infection rates under TNF inhibition and what does this imply for the individual patient? Ann Rheum Dis 2011;70:1914-20.

18. Bombardieri S, Ruiz AA, Fardellone P, Geusens P, McKenna F, Unnebrink K, et al. Effectiveness of adalimumab for rheumatoid 
arthritis in patients with a history of TNF-antagonist therapy in clinical practice. Rheumatology 2007;46:1191-9.

19. Emery P, Keystone E, Tony HP, Cantagrel A, van Vollenhoven R, Sanchez A, et al. IL-6 receptor inhibition with tocilizumab improves treatment outcomes in patients with rheumatoid arthritis refractory to anti-tumour necrosis factor biologicals: Results from a 24-week multicentre randomised placebo-controlled trial. Ann Rheum Dis 2008;67:1516-23.

20. Schiff M, Keiserman M, Codding C, Songcharoen S, Berman A, Nayiager $\mathrm{S}$, et al. Clinical response and tolerability to abatacept in patients with rheumatoid arthritis previously treated with infliximab or abatacept: Open-label extension of the ATTEST Study. Ann Rheum Dis 2011;70:2003-7.
21. Buch MH, Smolen JS, Betteridge N, Breedveld FC, Burmester G, Dörner T, et al. Updated consensus statement on the use of RTX in patients with rheumatoid arthritis. Ann Rheum Dis 2011;70:909-20.

22. Emery P, Mease PJ, Rubbert-Roth A, Curtis JR, Muller-Ladner U, Gaylis NB, et al. Retreatment with RTX based on a treatment-to-target approach provides better disease control than treatment as needed in patients with rheumatoid arthritis: A retrospective pooled analysis. Rheumatology 2011;50:2223-32. 